\title{
Téoros
}

Revue de recherche en tourisme

\section{Un Palais des congrès : to be or not to be}

\section{Claude Bédard}

Volume 4, numéro 3, novembre 1985

L'essor de l'industrie des congrès

URI : https://id.erudit.org/iderudit/1080790ar

DOI : https://doi.org/10.7202/1080790ar

Aller au sommaire du numéro

Éditeur(s)

Université du Québec à Montréal

ISSN

0712-8657 (imprimé)

1923-2705 (numérique)

Découvrir la revue

Citer cet article

Bédard, C. (1985). Un Palais des congrès : to be or not to be. Téoros, 4(3), 21-25.

https://doi.org/10.7202/1080790ar d'utilisation que vous pouvez consulter en ligne.

https://apropos.erudit.org/fr/usagers/politique-dutilisation/ 


\title{
Un Palais des congrès: to be or not to be
}

\author{
par Claude Bédard*
}

Y a-t-il des limites au développement d'une infrastructure touristique dans une région? A l'evidence, on ne peut répondre que par l'affirmative à cette question qui est souvent posée. II serait absurde, par exemple, de trouver à Montréal 200 grands hôtels ou encore que se tiennent consécutivement deux expositions universelles. En effet, le raisonnable ne touche pas seulement au bon sens mais aussi au portefeuille de chaque citoyen payeur de taxes engagé, parfois contre son gré, dans le développement

${ }^{*}$ Claude Bédard est vice-président Marketing a la Société du Palais des congres de Montréal de son industrie touristique. La lecture des journaux montréalais au cours des dernières années nous aident sans doute à poursuivre cette réflexion...

Voilà pourquoi l'État et les institutions, qui ont le contrôle sur les décisions d'engagement de capitaux pour de tels projets de développement, doivent savoir mesurer la limite entre ce qui pourrait s'appeler "l'effort politique" et les besoins réels d'une région en développement ou en consolida- tion de son infrastructure touristique.

Lors de l'édification du Palais des congrès de Montréal, la question a été posée très souvent. Montréal, déjà dotée d'une infrastructure hôtelière riche, capable de soutenir la demande en congrès, avait-elle vraiment besoin d'un Palais des congrès?

Allons plus loin: la présence d'un Palais des congrès allait-elle mettre en péril des institutions existantes, menaçant ainsi de

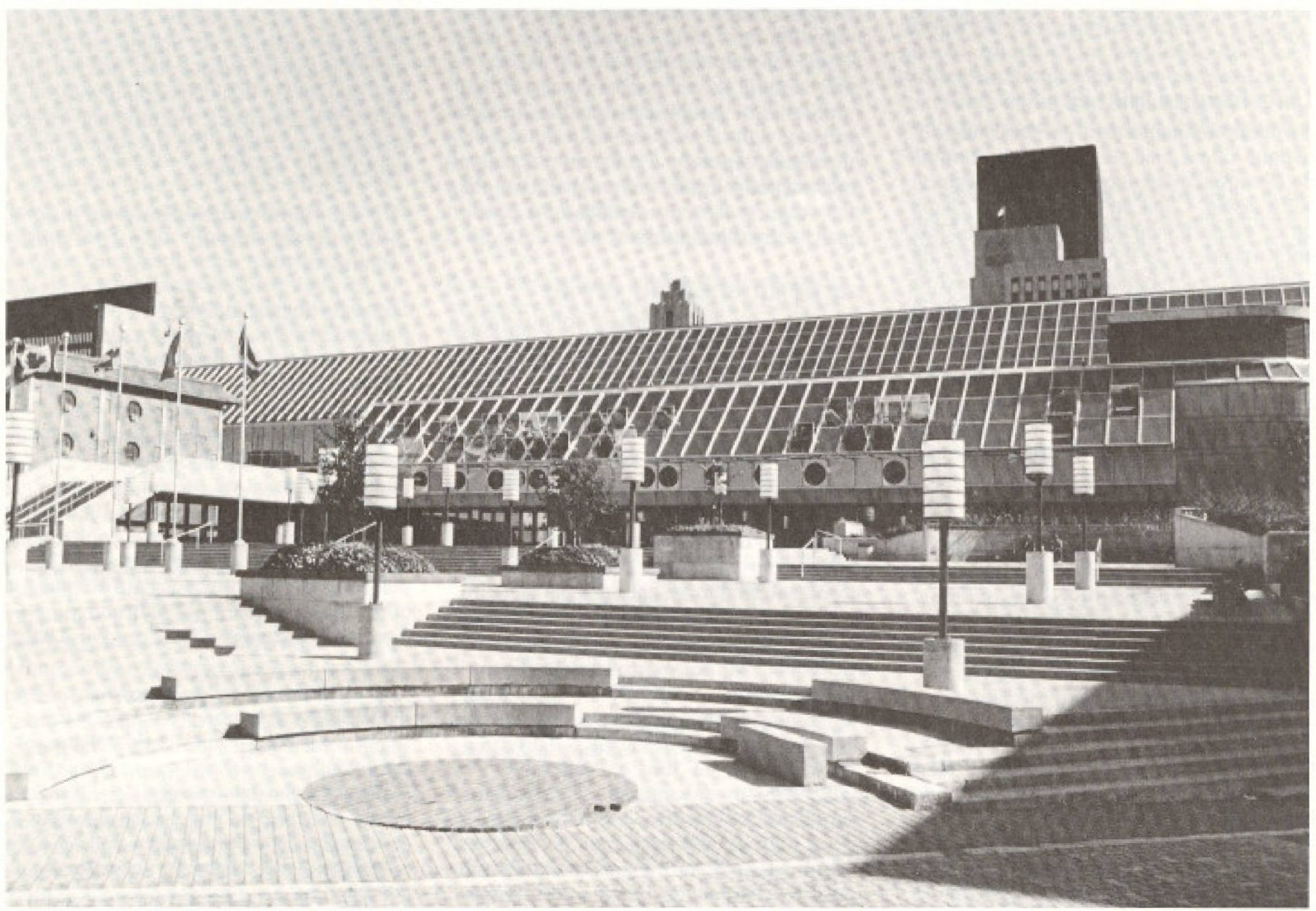

Ni le plus grand, ni le plus cher, le Palais des congrès de Montréal est une réponse réaliste pour équiper l'industrie touristique montréalaise d'une structure essentielle d'accueil. 
désuétude les grandes salles des hotels qui avaient jusqu'd̀ ce jour tenté de répondre à la demande des organisateurs de congrès?

Pour être juste, il faudrait insérer ces deux questions dans une autre encore plus large: Montréal se destinait-elle, au-delà de ses projets ponctuels tels qu'Expo 67, les Jeux Olympiques et les Floralies, à la difficile carrière internationale et se percevait-elle vraiment comme une grande métropole capable de concurrencer, sur certains aspects, Paris, New York ou Berlin.

En fait, Montréal avait-elle le désir de devenir un véritable carrefour international d'échanges d'idées, de projets et de réalisations?

Il est aisé de s'imaginer que devant de telles questions fondamentales, qui surviennent toujours lors d'injections importantes de capitaux, I'enfantement du Palais des congrés de Montréal n'a pas été facile et fut, à l'instar de plusieurs projets de ce type, I'occasion de l'exploitation des tensions deja existantes entre les divers intervenants dans le milieu. L'exemple du Palais des congrès de New York dont la construction fait l'objet de la chronique new yorkaise depuis les cinq dernières années démontre que le phénomène est le même partout où il est question d'investir dans ce domaine.

\section{Une longue histoire}

Symbole architectural de l'importance touristique d'une ville, le Palais des congres de Montréal a été évoqué dès le milieu du $\mathrm{XIXe}$ siècle. C'est en effet en 1841, alors qu'on préparait les fêtes marquant le $200 \mathrm{e}$ anniversaire de la fondation de la Ville de Montréal, qu'on parle pour la premiẻre fois d'un centre de congrès, c'est-ä-dire de la construction d'un "centre municipal".

Dans la premiẻre moitié du $\mathrm{XXe}$ siêcle, on a assisté à l'álaboration de plusieurs projets mais aucun n'a vu le jour. En 1935, on parle de construire un centre civique qui serait baptisé Léon-Trépanier.

L'idée est toutefois rapidement abandonnée. Un an plus tard, la Montreal Automobile Association fait exécuter les plans d'un édifice qu'elle se propose de construire sur la rue Bleury. Elle se heurte à un refus de la part des autorités de la ville de Montréal. Elle relance alors le projet en proposant le Carré Viger comme site, mais. encore lả, rien ne se concrétise.

En 1939, l'idée d'un centre civique refait surface et le Canadien National propose de l'aménager au-dessus de la Gare Centrale. Puis c'est la guerre, le projet se retrouve aux oubliettes. Un peu plus tard, de 1945 à 1950, le Conseil économique métropolitain élabore plusieurs projets dont aucun ne voit le jour.
Que s'est-il passé, entre les années 50 et 70 , pour qu'un tel projet aboutisse enfin? A l'évidence, l'évolution des mentalités, non seulement au Québec mais partout dans le monde, a joué un rôle essentiel.

On a d'abord assisté à de grandes migrations touristiques oủ les déplacements intercontinentaux devenaient chose commune et surtout accessible a tous les portefeuilles. Par ailleurs, le boom économique de l'après-guerre au Québec, la révolution tranquille, les différents projets d'envergure internationale qui ont vu le jour à Montréal ont amené celle-ci à réévaluer de façon positive cette question qui hantait les esprits depuis le milieu du XIXe siècle.

C'est donc en 1977 que le Conseil des ministres du Québec, â la suite d'une étude commandée au bureau d'ingénieursconseils Lavalin, décide de doter Montréal d'un Palais des congrès. En octobre 1979. c'est enfin la levée de la première pelletée de terre. Les travaux seront terminés en mai 1983.

A ceux qui s'objectaient à l'avènement d'un Palais des congrès a Montréal, invoquant l'argument classique que Montréal s'en était fort bien passée jusqu'à présent et que, de toute façon, les organisateurs de congrès se satisfaisaient largement des installations mises a leur disposition par les hótels (du reste assez bien pourvus), il était aisé de répondre que si Montréal voulait ne pas manquer le coche, comme cela s'était produit par le passé, elle devait sans plus tarder se doter d'un instrument quilui permettrait de répondre à la demande de plus en plus grande pour la tenue d'un congrês d'envergure dans des installations physiques, construites à cet effet.

\section{La pertinence du projet}

Une fois la décision prise et connue, plusieurs personnes ont soulevé la question de la pertinence d'un tel projet dans le développement économique de Montréal. Examinons ce point sous trois aspects précis:

\section{1) Le prix à paver en vaut-il les consé- quences dans le développement éco- nomique d'une ville ou d'un pays?}

L'évidence de la nécessité d'un Palais des congrés dans une ville à vocation internationale touristique est partagée par de nombreuses villes compétitrices. Seulement au Canada et aux Ėtats-Unis, il s'est construit récemment 40 nouveaux centres de congrès et encore Boston, New York, Baltimore. Washington et Philadelphie sont en voie d'en édifier de nouveaux. Toronto a ouvert le sien en 1984 et Vancouver en parachève un de dimension á peu prẻs semblable à celui de Montréal. Enfin, il existe plus de 200 Palais des congrès en Amérique du Nord et la plupart offrent, a quelques différences près, l'ensemble des services qu'offre le Palais des congres de Montréal.
Celui-ci a été construit en considération de la capacité hóteliẻre de Montréal. Ce n'est ni le plus grand, ni le plus coutteux; par exemple, Vancouver coutera 135 millions \$ selon les prévisions actuelles, et le Palais des congrès de Berlin a coûté 400 millions \$. Celui de New York dépassera bientôt le demi-milliard. En comparaison. Montréal a coûté 81 millions \$ et il est classé parmi les dix plus grands Palais des congres au monde.

Sur le plan budgétaire, il faut préciser que. par sa vocation, le Palais des congrês ne génèrera jamais de profits comme tels. Les quelques rares exceptions, dont la vocation principale est d'accueillir les congrès, qui réussissent à faire leurs frais dans le monde le font gếnéralement par des revenus en provenance de leurs activités périphériques comme, par exemple, des boutiques ou la location d'un terrain à un hôtel ou encore l'utilisation de leurs installations techniques par des entreprises extérieures qui font appel à eux chaque jour. En fait, l'efficacité financière d'un Palais des congres est établie en fonction des retombees qu'il génère dans le milieu économique et, principalement, touristique.

Ainsi, au cours du dernier exercice financier, les frais d'opération du Palais des congrès se sont élevés à environ 7 millions $\$$. En retour, il aura généré en 1984 des retombées économiques de quelques 103 millions $\$$ et percu directement des revenus de $3500000 \$ ; 909000$ personnes auront défilé dans ses murs, notamment 69800 étrangers, au cours des 146 événements qui s'y sont déroulés.

\section{2) Ya-t-il une complémentarité ou une concurrence avec /'industrie höte- liere?}

D'entrée de jeu, le Palais des congrès, à moins d'être trếs mal géré, contribue automatiquement á l'augmentation de l'industrie touristique d'une région. Bien appuyé par sa stratégie marketing, il n'aura de cesse d'amener chez lui une clientèle national et surtout internationale. Son activité dans les champs de sa compétence aura donc comme premier résultat d'attirer le plus de clients chez lui et par conséquent dans sa destination. A cet égard, un Palais des congres doit être consideré comme un outil supplémentaire qui agit comme élément moteur dans l'attraction d'une ville.

Afin de ne pas porter ombrage á son industrie hôtelière, le Palais des congrès de Montréal ne sollicite pas les congrẻs de moins de 1200 personnes qui pourraient être a I'aise, pour tenir leurs assises, dans certains hôtels de Montréal bien pourvus en salles de congrès. En s'imposant cette mesure restrictive dès le début de son existence, le Palais des congress de Montréal a voulu indiquer clairement son intention d'eviter d'enlever le marché traditionnellement des- 
tiné aux hôtels, pour se consacrer à une clientẻle-cible qui ne pouvait considérer Montréal comme destination à cause de ses exigences en termes d'espaces et de soutien logistique.

Il faut signaler que le Palais des congrès de Montréal n'abrite aucune des installations qui sont I'apanage des hôtels et qu'il n'est associé contractuellement à aucune chaîne hôtelière qui pourrait lui verser des redevances. II se considère comme étant au service de l'industrie hótelière toute entière et tient par toutes les mesures possibles à éviter de détoumer une clientèle déja fidèlé aux hôtels de Montréal. A titre d'illustration, non seulement il évite de démarcher ce qui revient aux hötels, mais en plus, il renvoie en moyenne une vingtaine d'évé. nements chaque mois au secteur hôtelier qu'il considère répondant aux critères de ses installations.

Jusqu'ici, et au risque de déplaire aux chercheurs de querelles, la très grande majoritế des clients du Palais des congrès de Montréal ne sont jamais venus auparavant à Montréal et, parmi les éléments influençant leur venue, le tandem Palais-Hôtels s'est averé un facteur déterminant.

II est concevable, compte tenu de sa jeunesse, que l'existence du Palais des congrès dérange le conformisme de certains qui étaient habitués à un style de marketing s'apparentant à une autre génération. De toute évidence, si on en juge par le bilan touristique des demieres années, les habitudes acquises n'avaient pas de toute façon donné les meilleurs résultats pour Montréal. L'arrivée du Palais des congrès dans le marketing congrès à Montreal changera-t-elle la situation? Pour l'heure, les premiers résultats s'avèrent fort encourageants. Déja, le nombre des evénements inscrits au calendrier du Palais des congrẻs est cinc fois plus élevé que ne l'avait prévu le rapport Laventhol and Horwath sur les retombés touristiques, publié en début 83 .

Parmi les éléments innovateurs dans la stratégie du marketing, il convient sans doute de souligner ici la création en mai 84 du comité des intervenants en congrès à Montréal (CICAM), qui regroupe les divers organismes engagés dans la sollicitation de congres.

Ces organismes sont l'Office des congres et du tourisme du Grand Montréal, la Com= mission d'initiative et de développement économiques de Montréal (CIDEM). l'Association des hôteliers de Montréal, Tourisme Québec, Tourisme Canada et la Société du Palais des congrès de Montréal. Ce regroupement des efforts a permis jusqu'ici d'atténuer les grandes difficultés qui pouvalent survenir entre les divers intervenants, de leur mieux faire comprendre la vocation, le rôle et les abjectifs de chacun en matière touristique. II a aussi permis d'éviter de faux débats sur I'hypothétique concurrence, crainte par l'entreprise privée soucieuse, avec raison, de rentabiliser ses installations. II a permis enfin d'aplanir un certain nombre de difficultés que rencontraient les clients désireux de choisir Montréal comme site de congrès.

En plus d'ouvrir la voie à la tenue de congrès plus imposants, en terme de nombre de délégués et de congressistes, et plus prestigieux, en regard de sa technologie d'avant-garde qui constitue l'un de ses atouts majeurs, la présence encore jeune du Palais des congrès de Montréal, au coeur de la ville, ne peut qu'avoir un effet benéfique sur l'ensemble de l'industrie touristique montréalaise.

Certains souligneront que les congressistes qui logent dans un hótel et qui tiennent leurs réunions au Palais laissent désespérément vides les salles de cet hôtel. II est vrai qu'à l'occasion, cette situation survienne, II s'agit la toutefois d'un faux problème.

D'une part, chaque hotel a le privilege de servir sa clientêle avant celle du Palais des congrès et par conséquent de réserver ses installations à des organisations qui logeraient chez lui et qui occuperaient ses salles de réunions. D'autre part, les congressistes des associations qui tiennent leurs réunions au Palais des congrès n'auraient tout simplement pas envisagé Montréal comme destination, si les installations physiques nécessaires n'avaient pas existé, et ne seraient donc pas venus. $\mathrm{Au}$ bout du compte, plus il y a de congressistes a Montréal et plus le chiffre des affaires de tous les intervenants augmente, plus il y a de retombées économiques.

Enfin, élèment non négligeable, les liens qui se tissent de jour en jour entre les intervenants depuis l'édification du Palais des congrès de Montréal, constituent un apport positif a son image. Des études de marché faites aux Etats-Unis à cet égard ont démontré déjả une meilleure perception de Montréal comme destination de congres. C'est justement à cause des liens privilégiés qu'ils entretiennent entre eux qu'on doit donc parler plutôt de complémentarité que de concurrence dans les rapports du Palais des congrẻs de Montréal avec l'industrie hótelière.

\section{3) Montreal est-elle veritablement une wille concurrente?}

Montreal a plusieurs atouts qui font d'elle une ville attrayante pour tenir un congres.

En raison de son patrimoine culturel et de sa particularité linguistique, Montréal présente un caractére européen tout en intégrant les caractéristiques nord-américaines. Montréal offre donc un certain dépaysement pour les Américains ou le reste du Canada, tout en conservant son cachet "américain" pour la clientèle outreAtlantique.

Elle dispose d'une excellente infrastructure hótelière d'un potentiel de 22000 chambres et des hótels de grande qualité. Montréal est aussi connue pour la richesse de sa table avec plus de 5000 restaurants. Une autre de ses particularités est sa ville souterraine qui permet une foule d'activités sans avoir ả sortir à l'extérieur, ce qui revêt une grande importance si I'on tient compte des rigueurs de l'hiver.

Les activités touristiques proprement dites sont nombreuses. Concerts, festivals, théatres, musées, cinémas, etc. sont légion au menu et les sites nombreux à visiter peuvent meubler des journées entiéres sans risque de lassitude.

Montréal est donc une ville de tourisme très active. De plus, elle est accessible grâce à une structure de transport très développée et sa proximité des grandes villes du nordest américain est un atout majeur dans le choix d'un site de congres.

Elle se devait, pour rester compétitive au niveau de lá concurrence, de posséder un outil aussi essentiel que le Palais des congrès.

Dans la compétition internationale, elle est maintenant en mesure de répondre aux exigences de plus en plus sérieuses que posent les associations internationales pour se réunir dans une ville car ses infrastructures sont à la hauteur des enjeux en cause.

Le Palais des congrès de Montréal, ni le plus cher, ni le plus grand, est une réponse réaliste pour équiper l'industrie touristique montréalaise d'une structure essentielle d'accueil qui s'avere finalement un atout majeure pour l'industrie hötelière du Grand Montréal. 


\section{Le centre de congrès: un concurrent nécessaire}

un témoignage de Georges Villedary

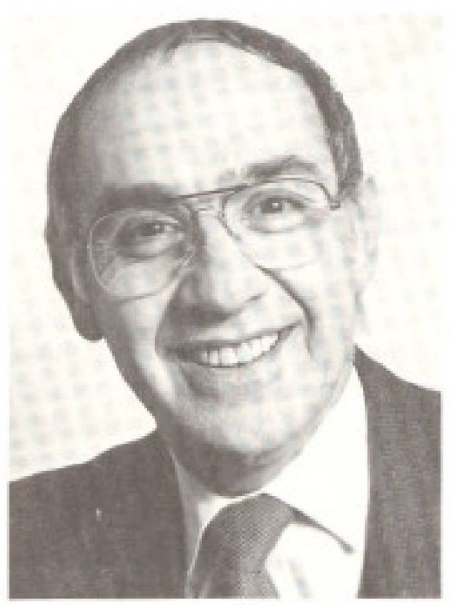

Monsieur Georges Villedary, directeur général du Centre Sheraton à Montréal.

M. Georges Villedary est directeur général du Centre Sheraton a Montréal. Comme directeur d'un hôtel important, il nous donne ici son avis sur l'importance des congrès et les rapports qu'entretiennent entre eux les hôtels et un centre de congrès.

A votre avis, quels sont les critères qui ont du poids dans la prise de décision pour un site de congres?

G.V.: Parfois des insfrastructures industriel- les, culturelles, sociales ou autres peuvent largement influencer le choix d'une ville. Malgré tout, je suis convaincu que seuls deux critères de base peuvent garantir l'obtention et ensuite le succès d'un congrès:s: lä réputation de la destination et la renommée des prestations de service hôtelier.

Une destination touristique telle que Montreal ou Québec attirera davantage de congressistes que "Trifouillis les oies". Même mieux, les participants seront enclins à se faire accompagner de leurs conjoints, charmes par la destination.

Souvent, ils décident de prolonger leur séjour pour jouer aux vrais touristes et visiter les alcntours. L'objectif est allors atteint; car nous avons réussi grâce à un congrề, à transformer notre participant avec sa famille en touriste, prolongeant ainsi un séjour, dépensant son argent dans notre destination et ses environs.

S'il est satisfait, il devient un ambassadeur, vantant les charmes et les qualités des endroits qu'il a visités, soit en congressiste, soit en touriste.

Il est indéniable que lors de la sélection d'une ville pour un congrès, les prestations hôtelières jouent un rôle primordial dans la décision finale. Les chaines d'hôtels à caractère international telles que Sheraton, Hilton,
Méridien, et j'en oublie quelques-unes car la liste serait trop longue, assurent à l'organisateur une exécution parfaite dans la préparation et dans le déroulement des activités du congres.

La présence d'un centre de congrès, près de votre etablissement, affecte-t-elle vos opérations?

G.V.: Il est indéniable que la présence d'un centre des congrès et la proximité de l'hótel par rapport au centre changent considerablement l'approche marketing.

Un hôtel localisé à proximite du centre des congres aura une politique de marketing toute à fait différente de celle d'un hôtel équipé pour recevoir des congrés mais dont l'emplacement nécessite un transport motorisé pour se rendre au centre des congrès.

Comment pouvez-vous concilier les besoins de tous vos clients avec ceux exprimés par lles congressistes?

G. V.: Pour un établissement hôtelier à vocation congrès, il est nécessalire de déterminer le nombre de chambres maximum que celuici va allouer aux congrès.

Une fois ce nombre déterminé, et ceci n'est pas facile car il faut considérer les différents segments de la clientẻle, les mois de l'annéc. les jours de semaine et enfin la régularité de la clientèle dite de passage, il est indispensable de considérer les differrentes sortes de congrès que l'hôtel peut accomoder.

J'aimerais ouvrir ici une parenthèse très breve

\section{Le Palais des congrès et la grande salle d'exposition de Place Bonaventure: d'abord et avant tout complémentaires}

une entrevue avec Jean L'Abbé

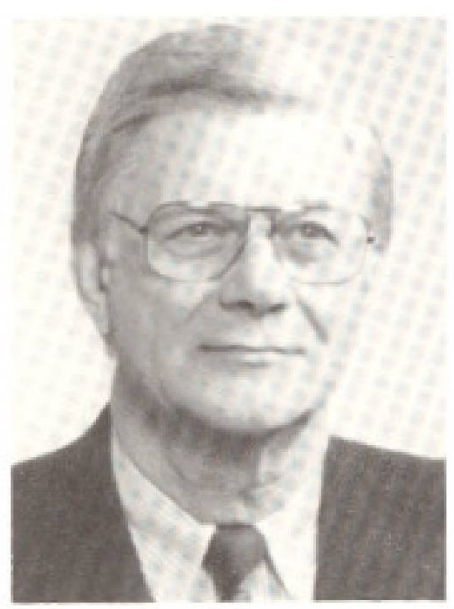

Monsieur Jean L'Abbé, directeur de la salle d'exposition de Place Bonaventure.
Depuis qu'elle a ouvert ses portes en 1967. la salle d'exposition de Place Bonaventure est toujours demeurée la plus grande et la plus complète au Canada. II y a longtemps qu'on a reconnu le rôle majeur qu'elle joue dans l'économie montréalaise qui a pu profiter de millions de dollars générés par les foires commerciales, les expositions et autres salons qui y ont été et y sont toujours présentés.

Cette année seulement, plus de 1,25 million de personnes devraient participer aux quelque 50 événements qu'on y tiendra.

M. Jean L'Abbé, directeur de la salle d'exposition, nous entretient des rapports entre la salle d'exposition de Place Bonaventure et le Palais des congrès.

Avec l'arrivée encore récente du Palais des congrès de Montréal, plusieurs se deman- dent si la salle d'exposition de Place Bonaventure a vu ses affaires péricliter?

J.L.: La plupart de ces curicux sont étonnés lorsqu'ils se font rểpondre qu'il n'en est rien, bien au contraire.

Le fait est que le Palais des congrès, qui est un apport indéniable pour Montréal, vient compléter l'ensemble de l'industrie hôtelière montréalaise, y compris la salle de Place Bonaventure. La raison en est bicn simple: les deux centres sont tout à fait différents.

Quelle est la vocation de chacun et leurs principales caractéristiques?

J.L.: La grande salle d'exposition reçoit un étalage de marchandises destinées à l'apprêciation d'un public d'acheteurs tandis que le grand centre de congress, lui, ne reçoit la plupart du temps que des rassemblements d'individus.

Lorsqu'on connait cette distinction fondamentale, il est plus facile d'apprécier ce qui différencie Place Bonaventure du Palais des congres. On comprend micux, par exemple, pourquoi ses salles d'exposition s'étendent sur 254000 pieds carrés contre 100000 pour le 
de commentaires entendus souvent par des. hommes d'affaires qui s' indignent qu'un hôtel réserve la totalité de ses chambres pour un congrès qui visite la destination une fois tous les cing ou dix ans, alors que eux, fréquentent l'établissement sur une base régulière de 40 a 45 semaines par an

Ces conmentaires très importants doivent çtre pris très sérieusement en consideration. Il faut cependant distinguer deux sortes de congrès de base.

\section{Quels sont-ils?}

\section{Le congrés whique}

Il occupe une grande partic ou la majorité des chambres de l'établissement, mais ne déborde pas dans d'autres hôtels. Il utilise toutes ou en partie les superficies commerciales à usage congrès de l'hôtel et de ce fait, devient le carburant financier le plus efficace. Les gestionnaires connaissent exactement les mouvements des congressistes et peuvent ainsi planifier à l'avance les equipements et le personnel nécessaires au fonctionnement de I'hotel.

C'est le genre de congrès recherché par un ćtablissement qui désire rentabiliser ses investissements dans les délais les plus courts.

Un autre avantage de ce genre de congrès se situe poư la chaîne d'hôtel qui peut suggérer des destinations avoisinantes pour des périodes avant ou apres le congres.

Le congrès à plusieurs hotels

C'est le genre de congres le plus difficile à qualifier, à prévoir ct qui, de plus, offre au centre des congrès sa clientèle au détriment des hôtels impliqués.

Un congrès de 3000 a 4000 personnes nécessite plusieurs hôtels, mais toutes les activités du congrès vont se dérouler au centre, done, les hôtels auront leurs chambres occupées mais les surfaces congrès demeurent vacantes.

Est-il avantageux pour vous malgré cela?

G. V .: Le centre devient done le concurrent des hôtels. Toutefois, le centre des congrès est une attraction positive à la destination; si le centre n'existait pas, le congrès ne pourrait avoir lieu.

Le dilemme est présent actuellement à Montréal, dû au fait que le Palais des congrès est la nouvelle attraction de notre ville.

Tous les intervenants sont d'accord sur un point: le Palais des congrès agit comme un aimant très puissant pour la destination et ses cffets de catalyseur's ne peuvent qu étre bếnefiques pour Montréal et le Québec tout entier.

Durant les premières années de son existence, le palais fait une concurrence locale mais normale, car il se doit pour survivie financièrement d'attirer un marché qu'un ou deux hôtels auraient pu s'accaparer facilement.

Quel rỏle complémentaire un centre de congrìs peut-il jouer pour favoriser le développement de notre industrie?

G. V .: La vocation du palais n'est pas, à mon avis, totalement réservée aux congrès. Tout au contraire, il existe des activitếs pouvant être organisées sur les lieux du palais qui peuvent attirer autant de participants que les congrès. sans pour autant nuire à l'exploitation normale de nos établissements hôteliers.

Je fais référence ici à des salons annuels, des foires de tous genres, agricoles, industrielles, électroniques, scientifiques, informatiques, etc. Au cours des années, ces salons ou foires doivent acquérir une réputation internationale de façon à attirer une clientèle nord-américaine. Les présentations se doivent d'être de qualitế de façon à concurrencer nos voisins du sud.

\section{En conclusion?}

G.V.: L'industrie du congres est une source de revenus financiers tres importants, non seulement pour l'hôtellerie en particulier mais également pour tous les commerces et institutions financières qui profitent de cette manne touristique, sans participer de très près à une collaboration de la promotion avec les secteurs reconnus. Le congressiste dépense son argent en congressiste, en touriste, en curieux, en collectionncur, en père ou mère de famille et également en client qui pourra se laisser séduire par une vitrine très attrayante.

Certes, le palais est en concurrence directe avec les hôtels lorsqu'il s'agit de prestations de services, mais il est également un apport bénéfique pour les congres utilisant plusieurs hôtels. Pour l'hôtelier, il s"agit de deffinir exactement sa participation quant au nombre de services à offrir aux congrès, car c'est de ce nombre que dépendra sa rentabilité.

\section{hall du Palais des congrès.}

Le Palais des congrès est conçu pour recevoir sans problème plusieurs congrès à la fois et peut digérer facilement des milliers de délćgués: salles de conférence de dimensions différentes en grand nombre, salles de banquet et de rassemblement, cafétéria moderne, etc...

Le hall d'exposition de Place Bonaventure est plutồt aménagé et équipé pour que la manipulation des quantités énormes de marchandises qu'on retrouve dans les grands salons se fasse sans anicroche et rapidement et que les exposants et organisateurs puissent travailler à leur aise: débarcadère moderne et facile d'accès, équipements mécaniques de montage spécialisés, bureaux pour les promoteurs, comptoirs de "fast-food" pour les visiteurs, etc...

\section{Autrement dit...}

J.L.: Les deux endroits, on le voit bien, ne visent pas le mẻme marché. Ils n'ont pas non plus les mêmes objectifs. Alors que Place Bonaventure est d'abord et avant tout une entreprise privée qui doit viser la rentabilité pour survivre, le Palais des congrès est une création gouvernementale qui cherche à atti- rer à Montréal les congressistes étrangers et l'argent qu'ils dépensent.

Quelque soit le lieu, le client doit assumer des frais. La politique tarifaire du Palais et de Place Bonaventure se compare-t-elle?

J.L.: Dans un tel contexte, ils est surprenant que les structures tarifaires des deux établissements soient sensiblement les mẻmes en ce qui concerne les grandes expositions. locales.

En effet, au Palais des congrc̀s aussi bien qu'à la Place Bonaventure les clients doivent louer l'espace dont ils ont besoin au pied carré et par jour d'occupation. Soit dit en passant, les tarifs de location sont très compétitifs comparativement aux établissements de mềne genre qu'ils soient canadiens ou américains.

Toutefois, pour les congrès internationaux, le Palais des congres peut souvent compter sur des subventions gouvernementales si le poisson qu'on veut pêcher est de taille. Et pourquoi pas, puisque les retombés économiques des rassemblements d'envergure profitent à beaucoup de monde, y compris au gouvernement qui recueille plus de taxes?

D'ailleurs, le président dı Palais des con- grès, M. Yves Michaud, disait dans une récente entrevue: "Nos prix de location sont tellement bas, que dans certains cas ils ne couvrent mème pas les coûts de l'électricité. Mais je prêvois que dans cinq ans, avec un déficit de 15 millions \$, nous engendrerons des revenus pour l'économie québécoise de quelque 250 millions \$."

\section{J.L.: Que demander de plus!}

En perspective, comment envisagez-vous l'avenir?

J.L. : Ensemble, les salles de Place Bonaventure et le Palais des congrès de Montré́al peuvent attirer les plus grandes expositions et les plus importants congrès au monde. Récemment, le congrès des Alccoliques anonvmes qui a réuni 48000 personnes a utilisé à fond les deux centres. Des événements de cette taille profitent beaucoup à l'économie montréalaise parce qu'ils génèrent une activité intense qui touche tous les hốtels, les restaurants et les magasins de la ville.

Congressiste, acheteurs et exposants peuvent converger sur Montréal par milliers maintenant que la ville compte sur des équipements de qualité capables d'abriter les grands événements internationaux. 